\title{
Radiologiekongress Nord 2018
}

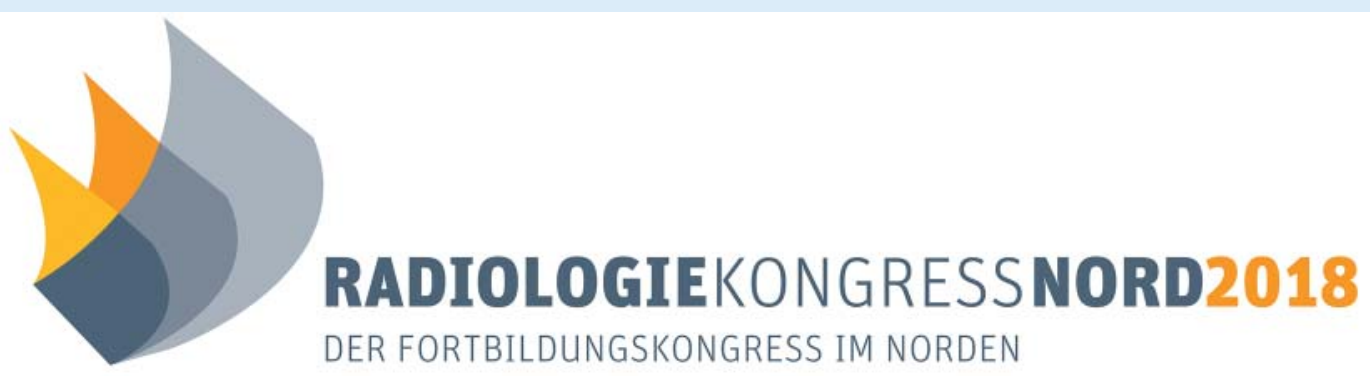

Am 16. und 17. Februar 2018 findet die gemeinsame Jahrestagung der Norddeutschen Röntgengesellschaft und der Röntgengesellschaft von Niedersachsen, Bremen und Sachsen-Anhalt im Radisson Blu Hotel Dammtor in Hamburg statt. Das Programm besticht durch seine Vielfältigkeit in den Themen und den Formaten. So können sich Ärzte und MTRA auf zahlreiche Workshop-Angebote freuen und auch für den radiologischen Nachwuchs wird es ein spezielles Programm geben. Im Folgenden finden Sie die Schwerpunkt-Themen sowie eine Auswahl der Highlights.

\section{Schwerpunkte und}

Highlights des

Radiologiekongresses

Nord 2018

\section{Panelrunde zum berufspolitischen} Thema „Radiologie - vier Perspektiven"

- Muskulo-Skelettal mit den großen 6: Schulter, Ellenbogen und Handgelenk, Hüfte, Knie und Sprunggelenk

- Interventionelle Radiologie - Leber und Gefäße

- Abdomen - Pankreas und Gefäße

- Urogenital - Erkrankungen der Prostata und Nierenerkrankungen

- Mamma - MRT, Früherkennung, Mammografie-Screening

- Strahlenschutz - neues Strahlenschutzgesetz, Dosismonitoring

- Varia - Diagnoseirrtum und/oder Diagnosefehler - juristische Unterschiede Neuroradiologie, Real-Time Kardio MRT und Vieles mehr

\section{Workshops (mit separater Anmeldung):}

- Nachweis des Prostatakarzinoms Freitag, 17:30 - 19:00 Uhr

- Workshop der Firma Bayer Vital GmbH „Lungenbildgebung - Aus dem radiologischen Alltag“ - Samstag, 08:30 12:30 Uhr

Workshop für Ärzte : Q1- und Q2-KardioCT-Kurs - Freitag, 08:30 - 19:00 Uhr
Workshops für MTRA in Kooperation mit dem Universitätsklinikum Eppendorf

Samstag, 08:30 - 11:00 Uhr

MTRA-Schülerprogramm ganztägig am Samstag, 08:30 - 16:00 Uhr

Alle Informationen zum Kongress und die Möglichkeit zur Anmeldung finden Sie auf www.roeko-nord.de

\section{Ihre Vorteile einer DRG-Mitgliedschaft}

Inhaltlich MITGESTALTEN

Aktive Beteiligung an der inhaltlichen Arbeit und Entwicklung des Fachs in Arbeitsgemeinschaften, Foren und Programmen.

Umfassend INFORMIEREN

Immer up to date durch Publikationen, Periodika und News-Services

Persönlich VERNETZEN

Fachlicher Austausch zu aktuellen Themen im Mitgliederbereich von drg.de, auf Veranstaltungen und Arbeitstreffen.

Bedarfsbezogen NUTZEN

Angebote und Leistungen in Anspruch nehmen wie z. B. die Zertifizierungsprogramme der DRG und ihrer Arbeitsgemeinschaften.

Praktisch ORGANISIEREN

Zusatzfunktionen und Services nutzen wie z. B. den individualisierten RöKo-Kongressplaner.

Finanziell PROFITIEREN Weniger bezahlen bei einer Teilnahme am Deutschen Röntgenkongress und weiteren Veranstaltungen der DRG, für Mitgliedschaften in internationalen Vereinigungen, den Bezug von Zeitschriften und für Produkte unserer Kooperationspartner. 